\title{
Identification of CALM as the potential serum biomarker for predicting the recurrence of nasopharyngeal carcinoma using a mass spectrometry-based comparative proteomic approach
}

\author{
HUILING MENG ${ }^{1,2}$, XIAODONG ZHU ${ }^{1,2}$, LING LI $^{1,2}$, ZHONGGUO LIANG ${ }^{1,2}$, \\ XIAOYU LI ${ }^{1,2}$, XINBIN PAN ${ }^{1,2}$, FANYAN ZENG ${ }^{1,2}$ and SONG QU ${ }^{1,2}$ \\ ${ }^{1}$ Department of Radiation Oncology, Affiliated Cancer Hospital of Guangxi Medical University, \\ Cancer Institute of Guangxi Zhuang Autonomous Region; ${ }^{2}$ Key Laboratory of High-Incidence-Tumor Prevention \\ and Treatment, Guangxi Medical University, Ministry of Education, Nanning, Guangxi 530021, P.R. China
}

Received June 27, 2016; Accepted July 31, 2017

DOI: $10.3892 / \mathrm{ijmm} .2017 .3094$

\begin{abstract}
To date, there are no serum biomarkers available for the prediction of recurrent nasopharyngeal carcinoma (rNPC). The diagnosis of rNPC mostly depends on imaging and biopsy of diseased tissue; however, both of these methods work mostly if the target tumor is at an advanced stage. Therefore, the identificaqtion of recurrent biomarkers is urgently required. In the present study, we used tandem mass tag (TMT) labeling and high performance liquid chromatography (HPLC) fractionation followed by liquid chromatography-tandem mass spectrometry (LC-MS/MS) to identify differentially expressed proteins. Serum was collected from 40 patients with NPC [recurrence $(n=20)$ and no recurrence $(n=20)]$. Compared to non-recurrent NPC (nrNPC), we found 59 proteins to be significantly dysregulated in rNPC; most of these have been previously reported to play a role in carcinogenesis. The dysregulation of calmodulin (CALM) was confirmed in 74 new patients [recurrence $(n=32)$ and no recurrence $(n=42)$ ] by ELISA. Moreover, we performed a preliminary pathway analysis which revealed that oxidative phosphorylation was altered in the patients with rNPC compared to those with nrNPC. Taken together, these data identify a potential diagnostic biomarker for rNPC and elucidate the potential molecular mechanisms that are dysregulated and contribute to the pathogenesis of rNPC.
\end{abstract}

\section{Introduction}

Nasopharyngeal carcinoma (NPC) is one of the most common types of cancer affecting populations in Southern

Correspondence to: Dr Song Qu, Department of Radiation Oncology, Affiliated Cancer Hospital of Guangxi Medical University, Cancer Institute of Guangxi Zhuang Autonomous Region, 71 Hedi Road, Nanning, Guangxi 530021, P.R. China

E-mail: 13607887386@163.com

Key words: recurrent nasopharyngeal carcinoma, calmodulin, tandem mass tags, biomarker
China andSoutheast Asia. It has led to a very serious health concern in these areas $(1,2)$. As far as we know, due to its location-specific characteristics and sensitivity to radiation, radiotherapy (RT) is the main treatment for NPC (3). With the appearance of intensity-modulated radiation therapy (IMRT), dose escalation, and the use of concurrent chemotherapy, the 5-year survival rate for patients with early-stage NPC can reach approximately $90 \%$, although for patients with advanced NPC, the rats is 70\% (4). However, approximately $20 \%$ of patients with NPC suffer from local recurrence even after the above-mentioned effective therapies (1). Currently, the detection of recurrent NPC (rNPC) mainly depends on the imaging or biopsy examination of diseased tissue; both of these methods work mostly if the target tumor is at an advanced stage (5). Furthermore, it is difficult to obtain tissue from recurrent disease for biopsy, and the difference between the side of recurrence and the tissue which changed by RT in imaging is difficult to distinguish. Therefore, the identification of serum biomarkers is warranted. In the first place, compared to tissue biopsy, the collection of serum is a less harmful method and serum is more easily obtained. Moreover, tumor biomarkers are molecules produced specifically or in excess of normal tissue by the pre-malignant cell, tumor cell or recurrent cancer cell, which may accumulate and are released into the circulation. These can help in the diagnosis, monitoring and prognosis of the treatment response (6). It is known that alphafetoprotein (AFP), cancer antigen (CA)-125, prostate-specific antigen (PSA) are used as serum biomarkers for the diagnosis, monitoring and the prognosis of liver, ovarian and prostate cancer, respectively. Serum biomarkers may also be used to identify recurrent cancers. At present, carcinoembryonic antigen (CEA) is a recurrent-related biomarker for colorectal tumors (7).

In addition, previous studies have reported some early predictive biomarkers for NPC, such as heat shock protein 27 (HSP27) (8), cathepsin D and keratin 8 (9), alpha-2 macroglobulin (A2M or AMG) and complement factor B (CFB) (10), as well as Galectin-1 (11). To date, some biomarkers for recurrent disease have also been reported, such as serum amyloid A (SAA) (12) and Epstein-Barr virus (EBV) 
DNA (13); however, these markers for rNPC still lack high predictive value in clinical practice. Therefore, there is an urgent need for the identification of novel putative biomarkers.

Furthermore, a preliminary pathway analysis is necessary, which can provide an approach with which to reveal the potential molecular mechanisms of recurrence, and which has been widely used in other diseases $(14,15)$.

In the present study, in an aim to identify new biomarkers for rNPC, and in order to analyze serum-expressed proteins, we performed tandem mass tag (TMT) labeling and high performance liquid chromatography (HPLC) fractionation followed by liquid chromatography-tandem mass spectrometry (LC-MS/MS). We considered that this would be an effective tool for screening proteomic biomarkers.

\section{Patients and methods}

Ethics. The present study was approved by the Joint Ethics Committee of the Guangxi Medical University Health Authority. All patients provided written informed consent prior to obtaining any samples. All human serum and clinical information was obtained from the Affiliated Cancer Hospital of Guangxi Medical University. According to the criteria established by the Chinese staging system of NPC in 2008 and the UICC/AJCC staging system in 2010; each patient with NPC was diagnosed and confirmed by a pathological examination.

Patient information. We selected 114 patients with NPC with histologically proven squamous cell carcinoma who were treated by IMRT or conventional radiotheraphy (con-RT) (a total prescribed dose of $\geq 70 \mathrm{~Gy}$ ), using a modified linear accelerator at the Affiliated Cancer Hospital of Guangxi Medical University, China, from January 2010 to June 2015. In total we recruited 52 patients with rNPC and 62 with non-recurrent NPC (nrNPC). The patients with rNPC were defined as those with histologically proven recurrence, and with no metastasis proven by imaging following radical cure RT 3 months later (with or without chemotherapy). Patients with nrNPC were those in which no proven recurrence or metastasis following radical cure RT (with or without chemotherapy) was found. The basic clinical parameters of the included patients are shown in Table I.

Serum separation. A total of $3 \mathrm{ml}$ venous blood was collected from each subject. Samples were placed standing for $30 \mathrm{~min}$ and then centrifuged at 3,000 rpm for $10 \mathrm{~min}$. The supernatant was separated and stored in different tubes at $-80^{\circ} \mathrm{C}$. A part of the serum was utilized for the discovery of dysregulated peptide, whereas the other part was prepared for the validation of target proteins.

Materials and reagents. Two pairs (termed studies; $10 \mathrm{rNPC}$ and $10 \mathrm{nrNPC} / \mathrm{study}$ ) patient sera were randomly selected and dissolved in a refrigerator at $2-8^{\circ} \mathrm{C}$. Subsequently, for reducing individual heterogeneity (16), an equal amount of 10 NPC samples from both the rNPC and nrNPC groups were mixed to generate 2 sample pools. Of the 20 Discovery samples (20 rNPC and 20 nrNPC) studyl randomly included $10 \mathrm{rNPC}$ and $10 \mathrm{nrNPC}$ samples; the remaining $10 \mathrm{rNPC}$ and 10 nrNPC samples, were included as study 2 .
Table I. Basic clinical parameters between patients with recurrent NPC, and those with non-recurrent NPC.

A, Discovery samples

\begin{tabular}{lccc}
\hline Variables & nrNPC & rNPC & P-value \\
\hline Sex (no.) & & & \\
$\quad$ Male & 15 & 16 & $>0.05$ \\
$\quad$ Female & 5 & 4 & $>0.05$ \\
Clinical stage (no.) & & & \\
Low (i+ii) & 5 & 2 & \\
High (iii+iv) & 15 & 18 & $>0.05$ \\
Median age (years) & 42 & 48.5 & $>0.05$ \\
\hline
\end{tabular}

B, Validation samples

\begin{tabular}{lccc}
\hline Variables & nrNPC & rNPC & P-value \\
\hline Sex (no.) & & & \\
$\quad$ Male & 35 & 29 & $>0.05$ \\
Female & 7 & 3 & $>0.05$ \\
Clinical stage (no.) & & 5 & \\
Low (i+ii) & 34 & 27 & $>0.05$ \\
High (iii+iv) & 45 & 46 & $>0.05$ \\
Median age (years) & & & \\
\hline
\end{tabular}

In all the parameters, no significant differences were observed between the patients with recurrent nasopharyngeal carcinoma (rNPC) and those with non-recurrent nasopharyngeal carcinoma (nrNPC).

High-abundance protein depletion. To enhance the accuracy of TMT analysis, high-abundance proteins were removed from the pooled samples using the ProteMiner (Protein Enrichment Large-Capacity kit, cat. no. 163-3006; Bio-Rad Laboratories, Inc., Hercules, CA, USA). The concentrated proteins were immunodepleted one more time. Finally, the protein was precipitated with cold $15 \%$ TCA for $2 \mathrm{~h}$ at $-20^{\circ} \mathrm{C}$. Following centrifugation at $20,000 \mathrm{xg}$ at $4^{\circ} \mathrm{C}$ for $10 \mathrm{~min}$, the supernatant was discarded. The remaining precipitate was washed with cold acetone 3 times. The protein was redissolved in buffer $(8 \mathrm{M}$ urea, $100 \mathrm{mM}$ TEAB, pH 8.0), and the protein concentration was determined with 2-D Quant kit (GE Healthcare Bio-Sciences, Pittsburgh, PA, USA). SDS-PAGE was used to evaluate the efficiency of high-abundance protein depletion in the two pooled samples (Fig. 1).

Trypsin digestion. For digestion, the protein solution was reduced with $10 \mathrm{mM}$ DTT for $1 \mathrm{~h}$ at $37^{\circ} \mathrm{C}$ and alkylated with 20 mM IAA (both from Sigma, St. Louis, MO, USA) for 45 min at room temperature in the dark. For trypsin digestion, the protein sample was diluted by the addition of $100 \mathrm{mM}$ TEAB to the urea concentration to $<2 \mathrm{M}$. Finally, trypsin was added at 1:50 trypsin-to-protein mass ratio for the first digestion overnight and 1:100 trypsin-to-protein mass ratio for a second $4 \mathrm{~h}$ digestion. Approximately $100 \mu \mathrm{g}$ protein for each sample was digested with trypsin for the following experiments. 


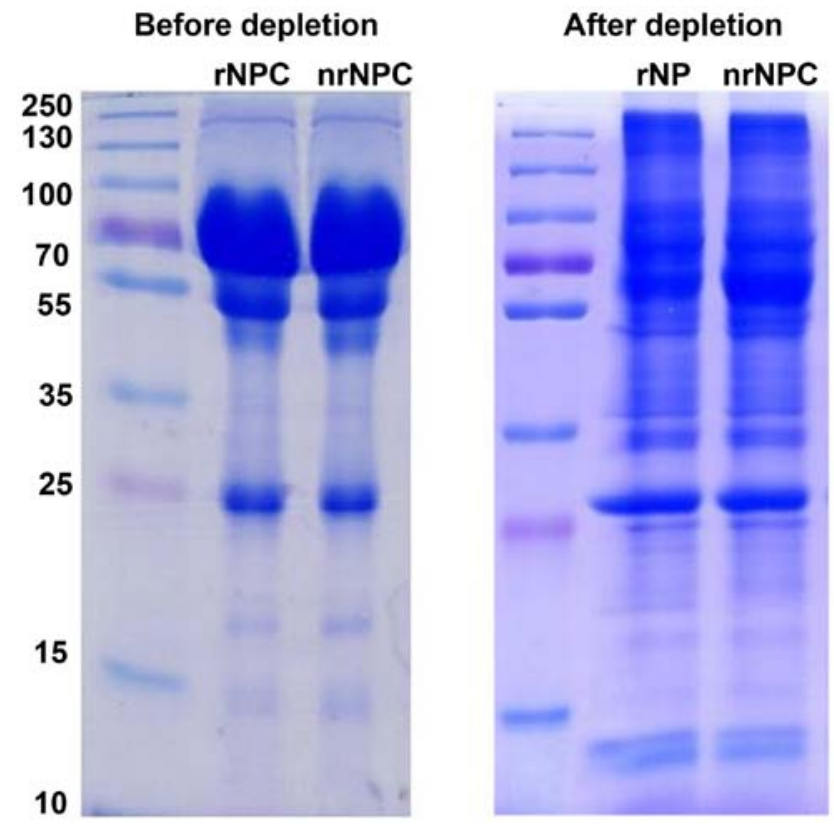

Figure 1. From SDS-PAGE, it is evident that high abundant protein was depleted well after depletion.

TMT labeling. Following trypsin digestion, for TMT labeling, peptide was desalted by a Strata-X C18 SPE column (Phenomenex, Inc., Torrance, CA, USA) and vacuum-dried. The peptide was reconstituted in $0.5 \mathrm{M}$ TEAB and processed according to the manufacturer's instructions provided with the 6-plex TMT kit (Thermo Fisher Scientific, Waltham, MA, USA). Briefly, one unit of TMT reagent (defined as the amount of reagent required to label $100 \mu \mathrm{g}$ of protein) was thawed and reconstituted in $24 \mu \mathrm{l}$ ACN (Thermo Fisher Scientific, Inc.). The peptide mixtures were then incubated for $2 \mathrm{~h}$ at room temperature and pooled, desalted and dried by vacuum centrifugation.

HPLC fractionation. The sample was then fractionated into fractions by high $\mathrm{pH}$ reverse-phase HPLC using an Agilent 300Extend-C18 column ( $5 \mu \mathrm{m}$ particles, $4.6 \mathrm{~mm}$ ID, $250 \mathrm{~mm}$ length; Agilent Technologies, Santa Clara, CA, USA). Briefly, the peptides were first separated with a gradient of 2-60\% acetonitrile in $10 \mathrm{mM}$ ammonium bicarbonate $\mathrm{pH} 10.0$ over $80 \mathrm{~min}$ into 80 fractions. The peptides were then combined into 18 fractions and dried by vacuum centrifugation.

LC-MS/MS analysis. The peptides were dissolved in $0.1 \%$ FA (Fluka, Buchs St. Gallen, Switzerland), directly loaded onto a reversed-phase pre-column (AcclaimPepMap 100; Thermo Fisher Scientific, Inc.). Peptide separation was performed using a reversed-phase analytical column (Acclaim PepMap RSLC; Thermo Fisher Scientific, Inc.). The gradient was comprised of an increase from 7 to $20 \%$ solvent $\mathrm{B}(0.1 \% \mathrm{FA}$ in $98 \% \mathrm{ACN})$ over $24 \mathrm{~min}, 20-35 \%$ in $8 \mathrm{~min}, 35-80 \%$ for $5 \mathrm{~min}$ and holding at $80 \%$ for the last $3 \mathrm{~min}$, all at a constant flow rate of $300 \mathrm{nl} / \mathrm{min}$ on an EASY-nLC 1000 UPLC system (Thermo Fisher Scientific, Inc.). The resulting peptides were analyzed by Q Exactive ${ }^{\mathrm{TM}}$ Hybrid Quadrupole-Orbitrap Mass Spectrometer (Thermo Fisher Scientific, Inc.).
The peptides were subjected to NSI source followed by tandem mass spectrometry (MS/MS) on a Q Exactive ${ }^{\mathrm{TM}}$ mass spectrometer (Thermo Fisher Scientific, Inc.) coupled online to the UPLC. Intact peptides were detected in the Orbitrap at a resolution of 70,000. Peptides were selected for MS/MS using NCE setting as 27, 30, 33; ion fragments were detected in the Orbitrap at a resolution of 17,500. A data-dependent procedure that alternated between one MS scan followed by $20 \mathrm{MS} / \mathrm{MS}$ scans was applied for the top 20 precursor ions above a threshold ion count of 1.0E4 in the MS survey scan with $30.0 \mathrm{sec}$ dynamic exclusion. The electrospray voltage applied was $2.0 \mathrm{kV}$. Automatic gain control was used to prevent overfilling of the ion trap; 5E4 ions were accumulated for generation of MS/MS spectra. For MS scans, the $\mathrm{m} / \mathrm{z}$ scan range was 350-1,800. Fixed first mass was set as $100 \mathrm{~m} / \mathrm{z}$.

Database search. The resulting MS/MS data were processed using Mascot search engine (v.2.3.0). Tandem mass spectra were searched against the Swiss-Prot Human Database (20,203 sequences). Trypsin/P was specified as cleavage enzyme allowing up to 2 missing cleavages. Mass error was set to $10 \mathrm{ppm}$ for precursor ions and $0.02 \mathrm{Da}$ for fragment ions. Carbamidomethyl on Cys, TMT-6 plex (N-term) and TMT-6plex $(\mathrm{K})$ were specified as fixed modification and oxidation on Met was specified as variable modifications. FDR was adjusted to $<1 \%$ and peptide ion score was set $>20$.

$Q C$ validation of MS data. Firstly, we checked the mass error of all the identified peptides. The distribution of mass error was near zero and most of them were $<0.02 \mathrm{Da}$, which meant that the mass accuracy of the MS data fit the requirement. Secondly, the length of most peptides was distributed between 8 and 16, which are in agreement with the property of tryptic peptides, which meant that sample preparation reached the standard (Fig. 2).

\section{Bioinformatics methods}

Gene Ontology (GO) analysis. All proteins identified in the serum samples were assigned a gene symbol using the UniProt-GOA database (http:www.ebi.ac.uk/GOA/). If some identified proteins were not annotated by UniProt-GOA database, the InterProScan software would be used to annotated protein's GO functional based on protein sequence alignment method. Protein classification was performed by GO annotation based on biological process, cellular component and molecular function. The differentially expressed proteins were further analyzed for pathway enrichment analysis by using Kyoto Encyclopedia of Genes and Genomes (KEGG) database. At the same time, we performed protein functional enrichment and performed a functional enrichment-based clustering for protein groups.

Selection of rNPC biomarkers. Dysregulated proteins were selected for further verification if they met one or more requirements: i) it should be a 'secreted' protein (14); ii) was shown to be involved on the KEGG database; and iii) the fold change of the protein was $\geq 1.2$ or $\leq 0.83$.

ELISA validation. Human ELISA kits were used for calmodulin (CALM; TSZ Bioscience, North Brunswick, NJ, USA), and experimental steps were performed as recommended by 

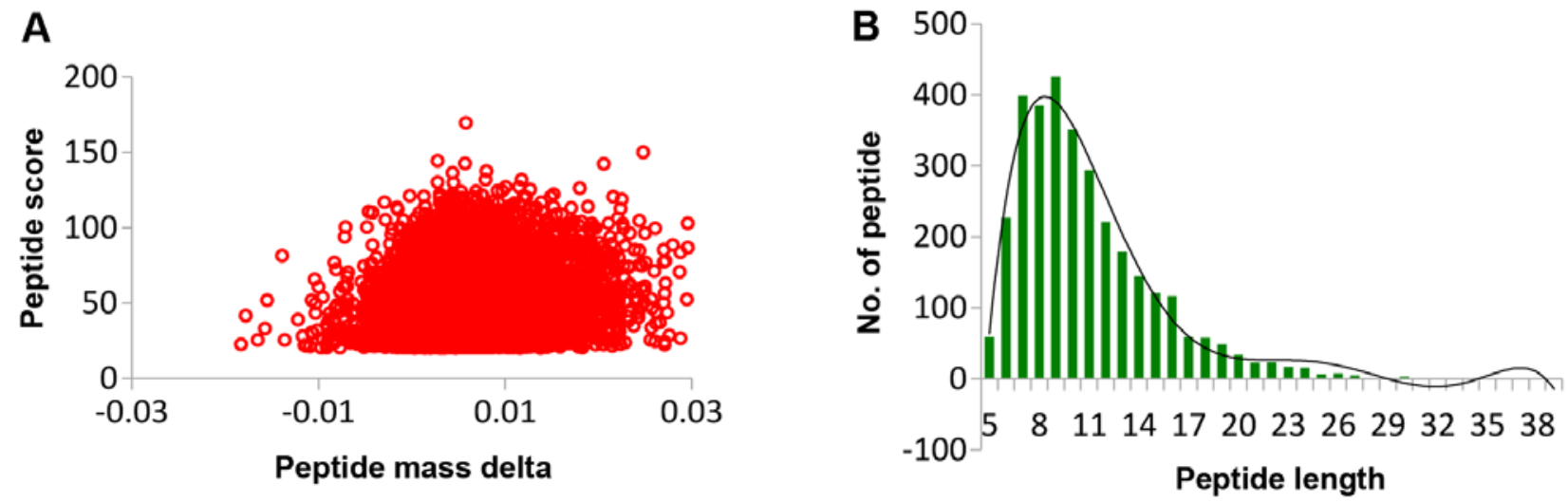

Figure 2. QC validation of MS data. (A) Mass error distribution of all identified peptides; (B) peptide length distribution. MS, mass spectrometry.

the manufacturer. Serum samples were analyzed for a total of 74 patients with NPC (32 rNPC +42 nrNPC).

Statistical analysis. All statistical analyses were performed using SPSS 21.0 software and GraphPad Prism 5.0 software. The differences between the two states of NPC were analyzed using a Mann-Whitney $U$ test. In addition, the target protein was evaluated for its capacity to distinguish between rNPC and nrNPC by making their ROC curves based on the ELISA scores. A value of $\mathrm{P}<0.05$ was considered to indicate a statistically significant difference.

\section{Results}

A schematic of the work flow is shown in Fig. 3. Using TMT labeling and HPLC fractionation followed by high-resolution LC-MS/MS analysis, we compared the 2 pooled NPC samples from the 10 patients with rNPC and the 10 patients with nrNPC (controls) in biological repeats. We indentified 635 protein groups from pooling human serum with a $99 \%$ confidence score and a $1 \%$ local FDR in each of the 2 analyzed groups, among which 413 proteins were quantified proteins, all were considered to be dysregulated if TMT ratios were $\geq 1.2$ or $\leq 0.83$ in $\geq 50 \%$ in rNPC relative to $n r N P C$; finally, dysregulated proteins were obtained in each group. The dysregulated proteins are shown in Table II and Fig. 4.

Functional classification of differentially quantified proteins. According to the GO annotation information of identified proteins, we calculated the number of differentially expressed proteins in each GO term of level 2. The upregulated proteins were main involved in the biological process of single-organism process (26/29), cellular process $(25 / 29)$, response to stimulus 20/29) etc, and the cellular component main included cell (23/29), organelle (21/29), macromolecular complex (13/29) etc. Molecular function mainly included binding (15/29), catalytic activity (11/29) etc. The details are shown in Table III.

The downregulated proteins were main involved in single-organism process (40/54), biological regulation (39/54), response to stimulus $(35 / 54)$ etc, the cellular component main included extracellular region (37/54), cell (34/54),

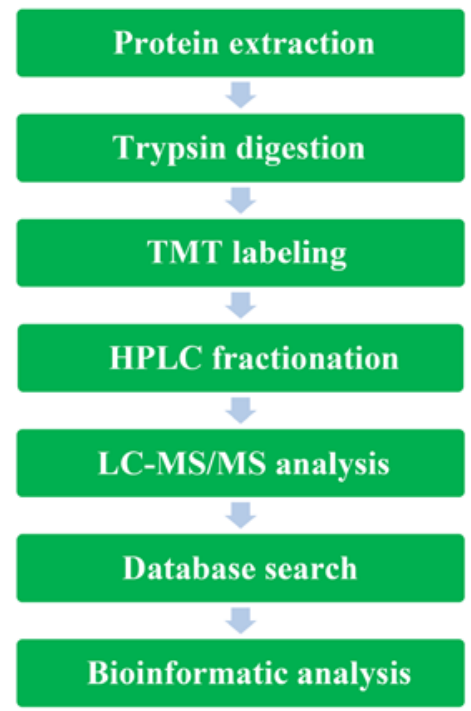

Figure 3. The aim of this study was to use an integrated approach involving TMT labeling, HPLC fractionation and mass spectrometry-based quantitative proteomics to quantify dynamic changes of the whole proteome of human serum. The general technical route is indicated. TMT, tandem mass tags; HPLC, high performance liquid chromatography.

membrane (20/54) etc. Molecular function main included binding (15/29), catalytic activity (11/29) etc. The details are shown in Table III.

GO-based enrichment analysis. We then we performed a GO-based enrichment analysis, molecular function included guanyl ribonucleotide binding, GTP binding etc, and most proteins were formed nucleosome, DNA bending complex, microtubule cytoskeleton etc. In addition, numerous proteins were involved in response to calcium, regulated cell cycle biological process etc. The details are shown in Fig. 5.

KEGG pathway enrichment. KEGG pathway enrichment included oxidative phosphorylation. According to KEGG pathway analysis, CALM was enriched in a significant pathway, and the enrichment ratio of CALM was the highest; moreover, the fold change of CALM was 2.11 (Fig. 6). Thus, we selected CALM to perform a further confirmed test. 
Table II. Differentially expressed and dysregulated proteins in nasopharyngeal carcinoma.

A, Summary of differentially quantified proteins $(>1.2$, or $<0.83)$

\begin{tabular}{lcr}
\hline Name & Upregulated $(>1.2)$ & Downregulated $(<0.83)$ \\
\hline Y1vsN1 & 29 & 54 \\
Y2vsN2 & 29 & 65 \\
YvsN & 28 & 47 \\
N1vsN2 & 0 & 1 \\
Y1vsY2 & 0 & 1 \\
\hline
\end{tabular}

B, Top 5 of upregulated proteins (Y1:rNPC, N1:rNPC)

\begin{tabular}{|c|c|c|c|c|c|c|c|c|c|}
\hline $\begin{array}{l}\text { Protein } \\
\text { accession no. }\end{array}$ & $\begin{array}{l}\text { Gene } \\
\text { name }\end{array}$ & Protein description & $\begin{array}{l}\text { MW } \\
\text { (Da) }\end{array}$ & $\mathrm{pI}$ & $\begin{array}{l}\text { AASC } \\
(\%)\end{array}$ & MP & Score & $\begin{array}{l}\mathrm{Y} 1 / \mathrm{N} 1 \\
\text { ratio }\end{array}$ & $\begin{array}{c}\text { Y1/N1 } \\
\text { P-value }\end{array}$ \\
\hline $\mathrm{P} 02741$ & CRP & C-reactive protein & 28,631 & 5.45 & 23.7 & 7 & 781 & 3.862 & $3.249 \mathrm{E}-12$ \\
\hline P13637 & ATP1A3 & $\begin{array}{l}\text { Sodium/potassium-transporting } \\
\text { ATPase subunit alpha-3 }\end{array}$ & 126,394 & 5.22 & 2.9 & 2 & 98 & 2.356 & 0 \\
\hline P02686 & MBP & Myelin basic protein & 37,681 & 9.79 & 7.2 & 2 & 78 & 2.26 & 0.030696 \\
\hline P62158 & CALM2 & Calmodulin & 18,889 & 4.09 & 16.1 & 3 & 40 & 2.11 & 0.019282 \\
\hline P60174 & TPI1 & Triosephosphate isomerase & 35,869 & 5.65 & 7 & 2 & 49 & 2.045 & 0.127868 \\
\hline
\end{tabular}

C, Top 5 of downregulated proteins

\begin{tabular}{|c|c|c|c|c|c|c|c|c|}
\hline $\begin{array}{l}\text { Protein } \\
\text { accession no. }\end{array}$ & $\begin{array}{l}\text { Gene } \\
\text { name }\end{array}$ & Protein description & $\mathrm{pI}$ & $\begin{array}{c}\text { AASC } \\
(\%)\end{array}$ & MP & Score & $\begin{array}{c}\mathrm{Y} 1 / \mathrm{N} 1 \\
\text { ratio }\end{array}$ & $\begin{array}{c}\text { Y1/N1 } \\
\text { P-value }\end{array}$ \\
\hline $\mathrm{P} 25705$ & ATP5A1 & ATP synthase subunit alpha, mitochondrial66,932 & 9.16 & 12.1 & 6 & 200 & 0.280 & $8.69 \mathrm{E}-06$ \\
\hline Q99798 & $\mathrm{ACO} 2$ & Aconitate hydratase, mitochondrial & 7.36 & 2.2 & 2 & 64 & 0.329 & 0.036558 \\
\hline P06576 & ATP5B & ATP synthase subunit beta, mitochondrial62,025 & 5.26 & 12.9 & 5 & 170 & 0.339 & 0.003399 \\
\hline P09972 & ALDOC & Fructose-bisphosphate aldolase C & 6.41 & 10.2 & 2 & 129 & 0.347 & 0.2142 \\
\hline P11678 & EPX & Eosinophil peroxidase & 10.31 & 7.6 & 4 & 99 & 0.408 & 0.038348 \\
\hline P61160 & ACTR2 & Actin-related protein 2 & 6.3 & 4.6 & 2 & 91 & 0.501 & 0.148856 \\
\hline
\end{tabular}

D, All dysregulated proteins

\begin{tabular}{|c|c|c|c|}
\hline $\begin{array}{l}\text { Protein } \\
\text { accession no. }\end{array}$ & Protein description & $\mathrm{Y} / \mathrm{N}$ ratio & $\begin{array}{l}\text { Regulated } \\
\text { type }\end{array}$ \\
\hline P02741 & $\mathrm{C}$-reactive protein & 3.862 & Up \\
\hline Q9BXR6 & Complement factor H-related protein 5 & 1.433 & Up \\
\hline P62158 & Calmodulin & 2.11 & $\mathrm{Up}$ \\
\hline P67936 & Tropomyosin alpha-4 chain & 1.464 & Up \\
\hline O43852 & Calumenin & 1.361 & Up \\
\hline P04211 & Ig lamda chain $\mathrm{V}$ region $4 \mathrm{~A}$ & 1.517 & Up \\
\hline P02743 & Serum amyloid P-component & 1.295 & Up \\
\hline P20742 & Pregnancy zone protein & 1.208 & Up \\
\hline Q15485 & Ficolin-2 & 1.502 & $\mathrm{Up}$ \\
\hline P0DJI9 & Serum amyloid A-2 protein & 2.036 & Up \\
\hline P02671 & Fibrinogen $\alpha$ chain & 1.217 & Up \\
\hline P01598 & Ig kappa chain V-I region EU & 1.507 & Up \\
\hline Q9HBI1 & Beta-parvin & 1.638 & Up \\
\hline Q9Y490 & Talin-1 & 1.521 & $\mathrm{Up}$ \\
\hline Q13201 & Multimerin-1 & 1.238 & Up \\
\hline Q08830 & Fibrinogen-like protein 1 & 1.361 & Up \\
\hline
\end{tabular}


Table II. Continued.

\begin{tabular}{|c|c|c|c|}
\hline $\begin{array}{l}\text { Protein } \\
\text { accession no. }\end{array}$ & Protein description & $\mathrm{Y} / \mathrm{N}$ ratio & $\begin{array}{l}\text { Regulated } \\
\text { type }\end{array}$ \\
\hline P02686 & Myelin basic protein & 2.26 & Up \\
\hline P35542 & Serum amyloid A-4 protein & 1.494 & Up \\
\hline P05155 & Plasma protease $\mathrm{C} 1$ inhibitor & 0.763 & Down \\
\hline P80748 & Ig lamda chain V-III region LOI & 0.772 & Down \\
\hline P05534 & HLA class I histocompatibility antigen, A-24 $\alpha$ chain & 0.545 & Down \\
\hline O75882 & Attractin & 0.616 & Down \\
\hline P36955 & Pigment epithelium-derived factor & 0.721 & Down \\
\hline P06314 & Ig kappa chain V-IV region B17 & 0.759 & Down \\
\hline Q5QNW6 & Histone H2B type 2-F & 0.693 & Down \\
\hline P01597 & Ig kappa chain V-I region DEE & 0.747 & Down \\
\hline P04217 & Alpha-1B-glycoprotein & 0.729 & Down \\
\hline Q16610 & Extracellular matrix protein 1 & 0.675 & Down \\
\hline P06312 & Ig kappa chain V-IV region (fragment) & 0.746 & Down \\
\hline P25705 & ATP synthase subunit alpha, mitochondrial & 0.28 & Down \\
\hline P25311 & Zinc-alpha-2-glycoprotein & 0.678 & Down \\
\hline P23083 & Ig heavy chain V-I region V35 & 0.687 & Down \\
\hline P01009 & Alpha-1-antitrypsin & 0.78 & Down \\
\hline P01767 & Ig heavy chain V-III region BUT & 0.671 & Down \\
\hline P08571 & Monocyte differentiation antigen CD14 & 0.58 & Down \\
\hline P06576 & ATP synthase subunit $\beta$, mitochondrial & 0.339 & Down \\
\hline P01042 & Kininogen-1 & 0.817 & Down \\
\hline P01859 & Ig gamma-2 chain $\mathrm{C}$ region & 0.765 & Down \\
\hline P01860 & Ig gamma-3 chain $\mathrm{C}$ region & 0.766 & Down \\
\hline P32119 & Peroxiredoxin-2 & 0.642 & Down \\
\hline Q99798 & Aconitate hydratase, mitochondrial & 0.329 & Down \\
\hline P01034 & Cystatin-C & 0.769 & Down \\
\hline P01593 & Ig $\kappa$ chain $\mathrm{V}$-I region $\mathrm{AG}$ & 0.535 & Down \\
\hline P01834 & Ig $\kappa$ chain $\mathrm{C}$ region & 0.789 & Down \\
\hline P01615 & Ig $\kappa$ chain V-II region FR & 0.785 & Down \\
\hline P01781 & Ig heavy chain V-III region GAL & 0.77 & Down \\
\hline P49747 & Cartilage oligomeric matrix protein & 0.659 & Down \\
\hline B9A064 & Immunoglobulin lamda-like polypeptide 5 & 0.778 & Down \\
\hline P01611 & Ig kappa chain V-I region Wes & 0.755 & Down \\
\hline P00488 & Coagulation factor XIII A chain & 0.764 & Down \\
\hline P68871 & Hemoglobin subunit beta & 0.566 & Down \\
\hline P02790 & Hemopexin & 0.606 & Down \\
\hline P00738 & Haptoglobin & 0.818 & Down \\
\hline P06396 & Gelsolin & 0.711 & Down \\
\hline P62805 & Histone $\mathrm{H} 4$ & 0.766 & Down \\
\hline P01625 & Ig kappa chain V-IV region Len & 0.755 & Down \\
\hline $\mathrm{P} 02763$ & Alpha-1-acid glycoprotein 1 & 0.757 & Down \\
\hline P01768 & Ig heavy chain V-III region CAM & 0.64 & Down \\
\hline P05546 & Heparin cofactor 2 & 0.754 & Down \\
\hline
\end{tabular}

Y represents recurrent nasopharyngeal carcinoma (rNPC); N represents non-recurrent nasopharyngeal carcinoma (nrNPC).

Clustering analysis. KEGG pathway enrichment-based clustering demonstrated that the dysregulated proteins mainly involved oxidative phosphorylation etc; the details are shown in Fig. 7. Functional enrichment-based clustering revealed that the dysregulated proteins were mainly involved in responding to calcium ion, oxidative stress, transport and secrection. The details are shown in Fig. 8.

ELISA results. We performed a preliminary analysis to assess the potential value of CALM as a biomarker in serum from 
Table III. The GO terms of level 2 distribution of the proteins of $\mathrm{Y} / \mathrm{N}$

No.

GO terms level 1

GO terms level 2

of protein

Upregulated proteins

Biological process

Single-organism process

26

Cellular process

25

Response to stimulus

Biological regulation

Localization

Metabolic process

Signaling

Multicellular organismal

process

Cellular component

organization or biogenesis

Developmental process

Immune system process

Locomotion

Multi-organism process

Other

Cellular component Cell

Organelle

Macromolecular complex

Membrane

Extracellular region

Membrane-enclosed lumen

Cell junction

Other

Molecular function

Binding

Catalytic activity

Structural molecule activity

Enzyme regulator activity

Electron carrier activity

Downregulated

proteins

Biological process

e-organism process

Biological regulation

Response to stimulus

Metabolic process

Cellular process

Multicellular organismal

process

Immune system process

Localization

Developmental process

Cellular component

organization or biogenesis

Multi-organism process

Other

Cellular component Extracellular region

Cell

Membrane

Organelle
Table III. Continued.

No

\begin{tabular}{llr} 
GO terms level 1 & \multicolumn{1}{c}{ GO terms level 2 } & of protein \\
\hline & Macromolecular complex & 13 \\
& Membrane-enclosed lumen & 11 \\
& Extracellular matrix & 5 \\
& Other & 2
\end{tabular}

GO, Gene Ontology. Y represents recurrent nasopharyngeal carcinoma (rNPC); $\mathrm{N}$ represents non-recurrent nasopharyngeal carcinoma (nrNPC).
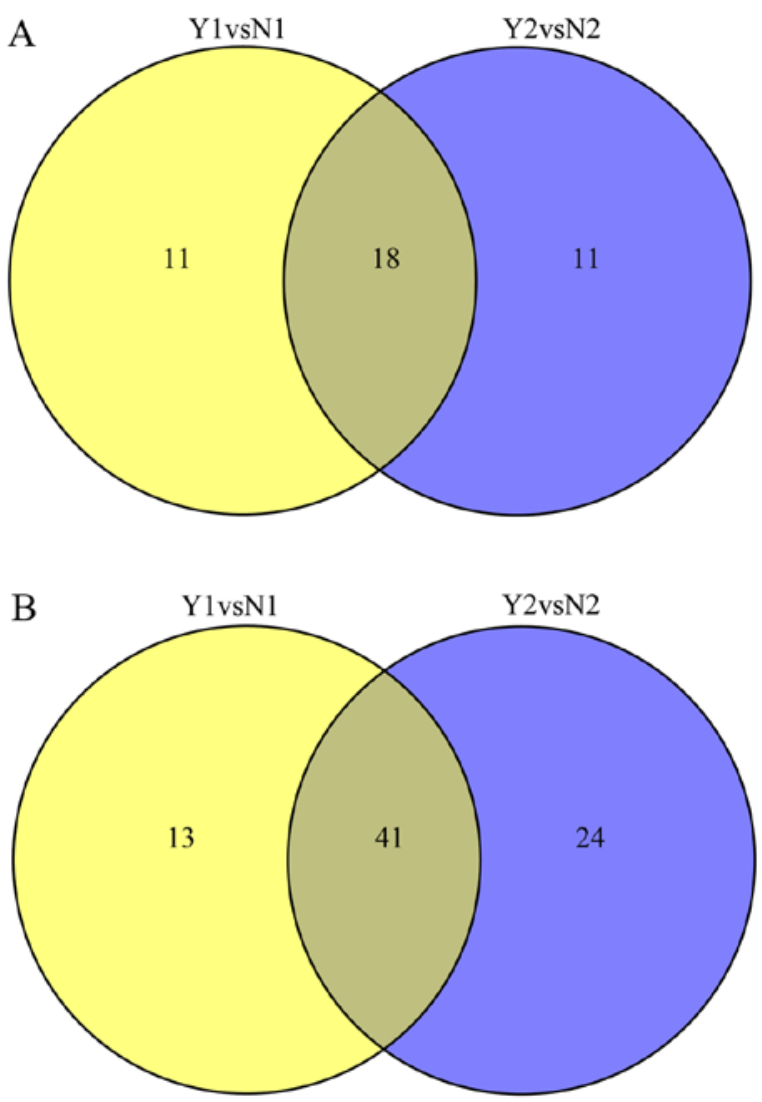

Figure 4. Venn diagrams showing the number of (A) upregulated and (B) downregulated protein identifications and identification overlap3 of study 1 and 2 . $\mathrm{Y}$ represents recurrent nasopharyngeal carcinoma (rNPC); N represents nonrecurrent nasopharyngeal carcinoma (nrNPC).

patients with rNPC. We found a significant difference between patients with rNPC compared to those with nrNPC (mean \pm SD) $(237.1 \pm 307 \mathrm{ng} / \mathrm{l}$ vs. $104 \pm 115.3 \mathrm{ng} / \mathrm{l})$, respectively $(\mathrm{P}=0.0233$, $\mathrm{P}<0.05$; Fig. 9) in the levels of serum, which was in agreement with our MS results. Furthermore, we performed a ROC curve analysis to evaluate the predictive value of serum CALM; the area under the ROC curve was 0.6931 (95\% CI: $0.5419-0.8443, \mathrm{P}=0.02275, \mathrm{P}<0.05)$. In addition, we have to point out that the total number in our sample was 74 patients (32 rNPC $+42 \mathrm{nrNPC}$ ), for the reason that some samples contained a low concentration which was beyond the reach of the ELISA kit; the concentration of CALM was only found 
A

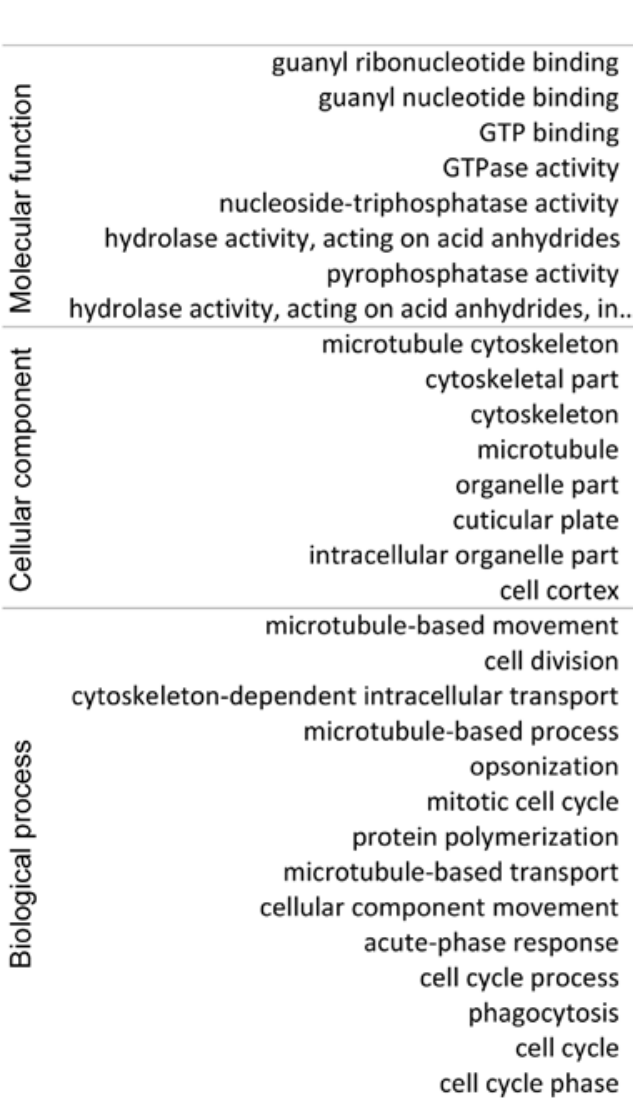

B

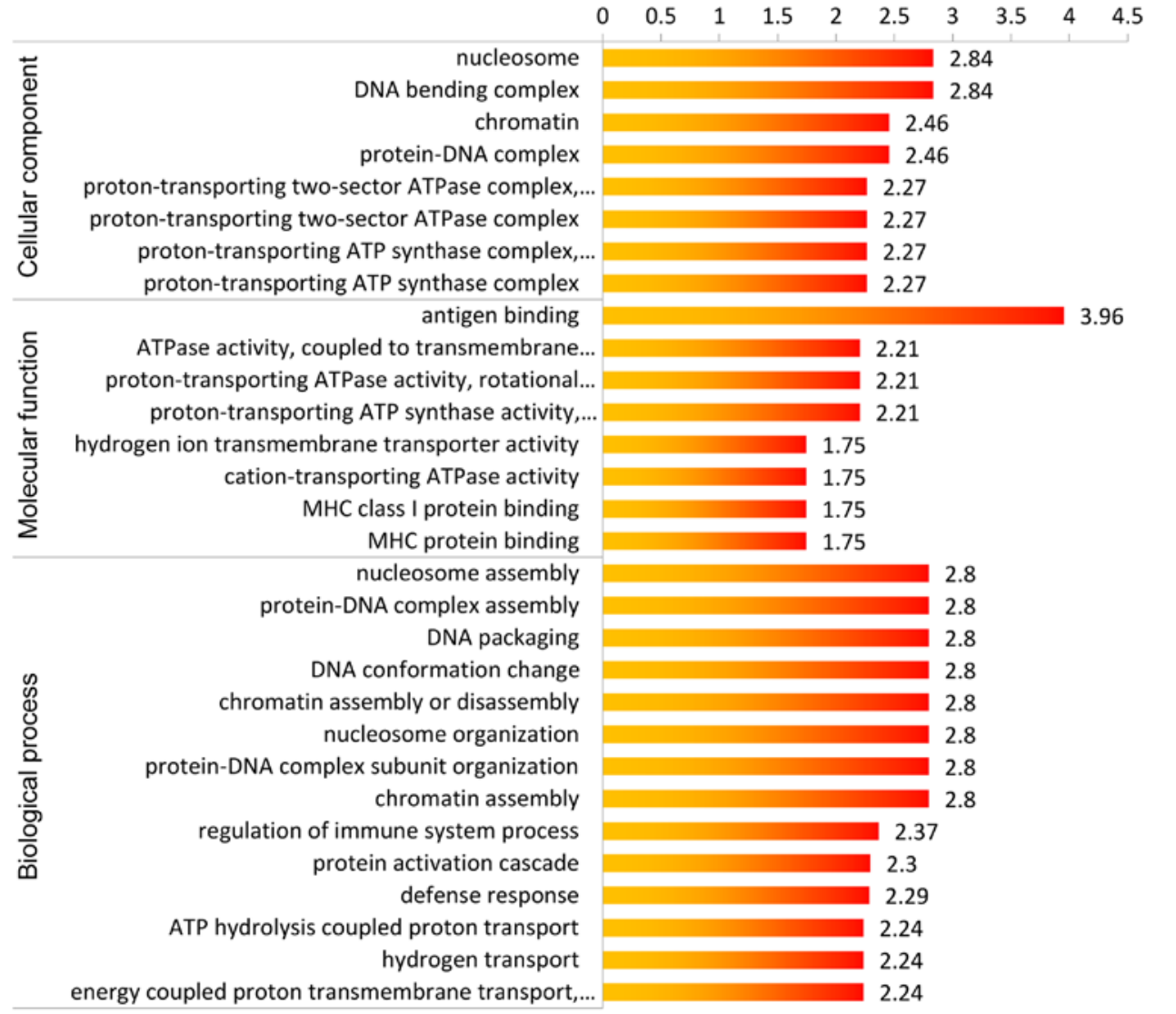

-log10(Fisher' exact test P-value)

$\begin{array}{lllllllllll}0 & 0.5 & 1 & 1.5 & 2 & 2.5 & 3 & 3.5 & 4 & 4.5 & 5\end{array}$

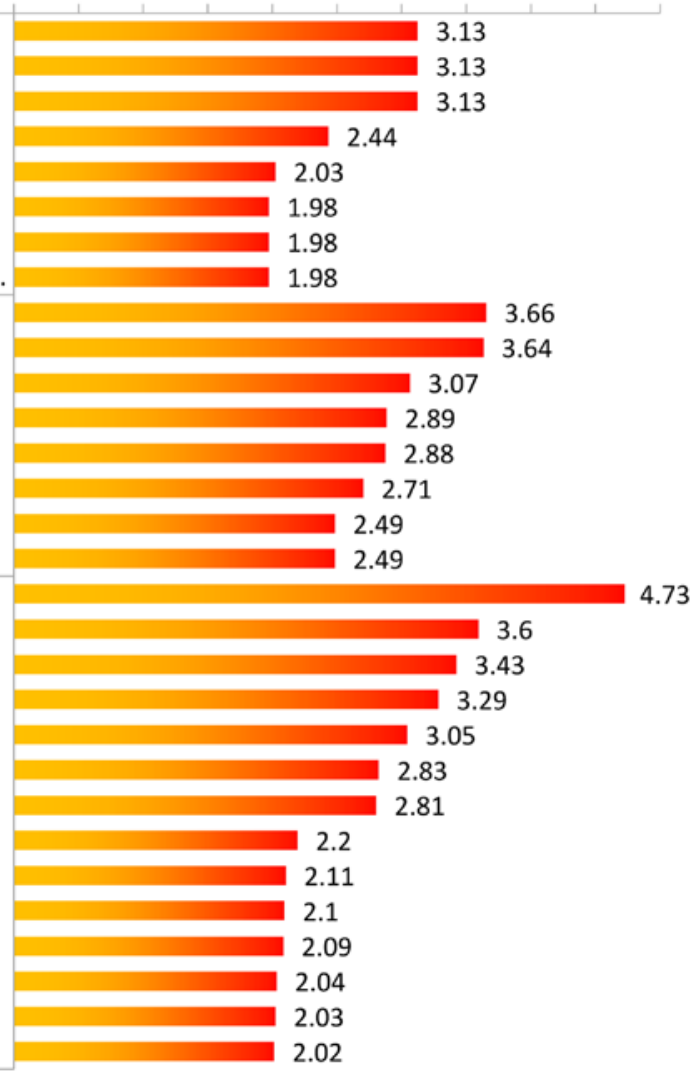

-log10(Fisher' exact test P-value)

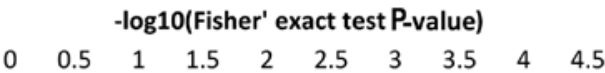

$\begin{array}{lll}0 & 0.5 & 1\end{array}$

Figure 5. (A) GO-based enrichment analysis of upregulated proteins, which shows guanyl ribonucleotide binding, guanyl nucleotide binding, GTP binding were the highest enrichment in cellular component, microtubule cytoskeleton, cytoskeletal part were the highest enrichment in molecular function, microtubule-based movement was the highest enrichment in biological process. (B) GO-based enrichment analysis of downregulated proteins, which shows nucleosome and DNA bending complex were the highest enrichment in cellular component, and antigen binding was the highest enrichment in molecular function, and nucleosome assembly protein-DNA complex assembly, DNA packaging were the highest enrichment in biological process. GO, Gene Ontology. 


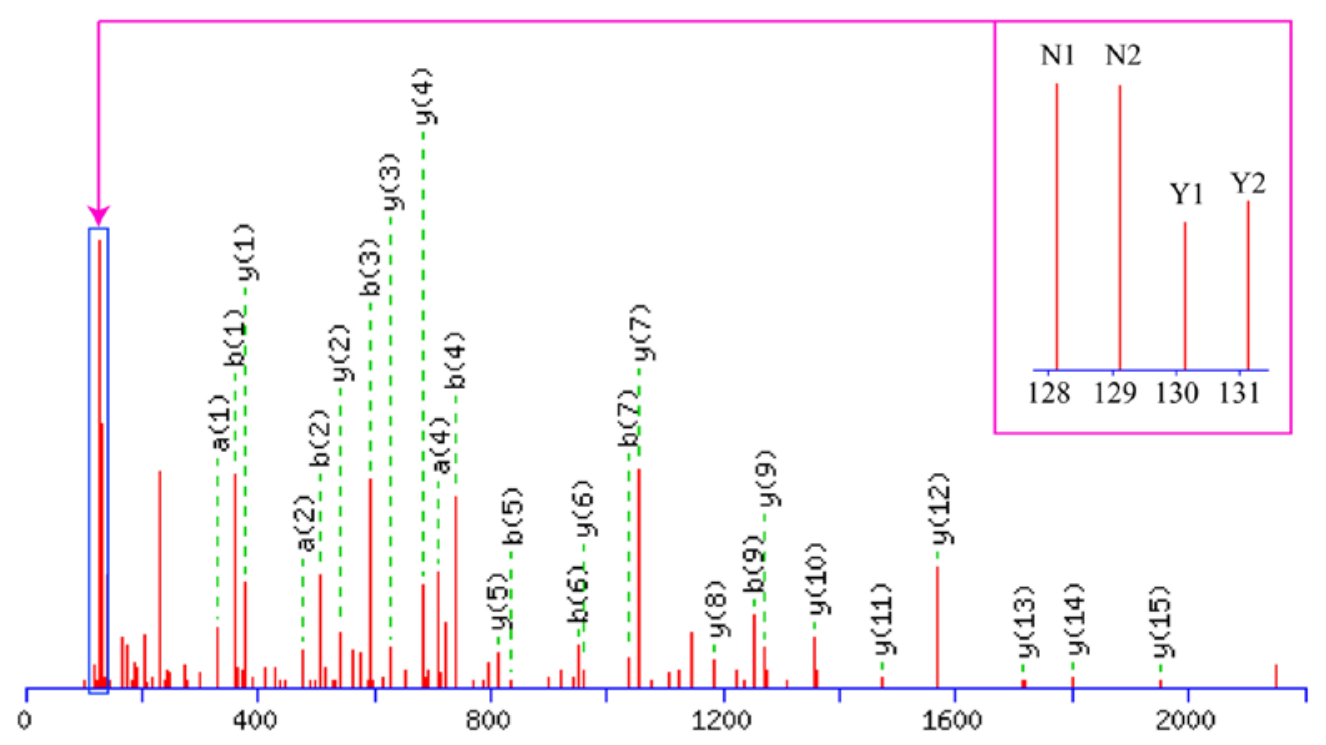

Figure 6. MS/MS spectrum of CALM. MS/MS, tandem mass spectrometry; CALM, calmodulin.

\section{KEGG pathway}

\section{Downregulated}

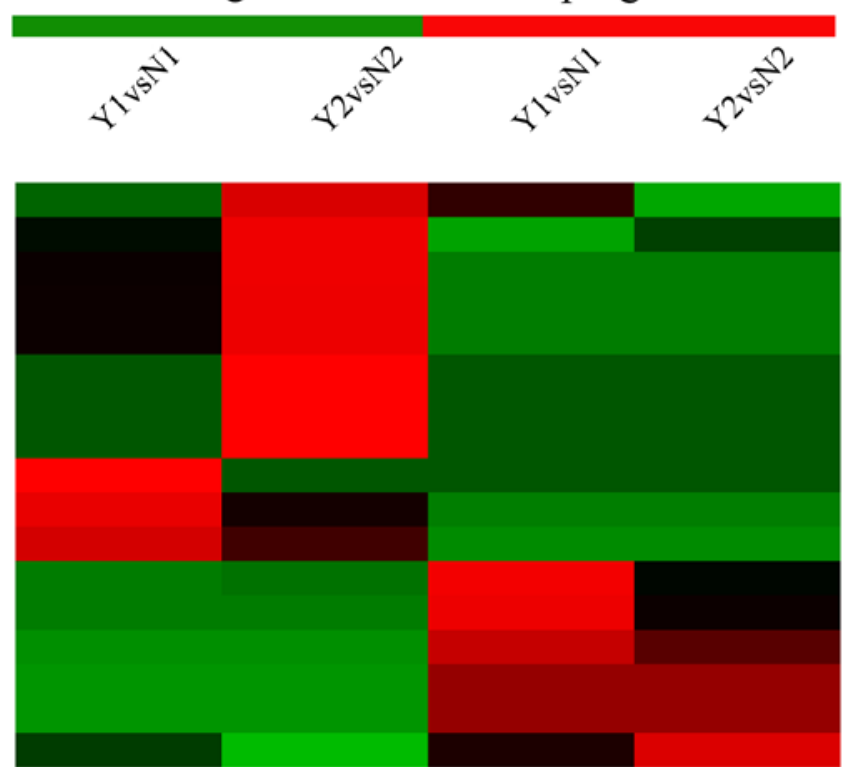

Zscore [- $\log 10$ (Fisher's exact P-value)]

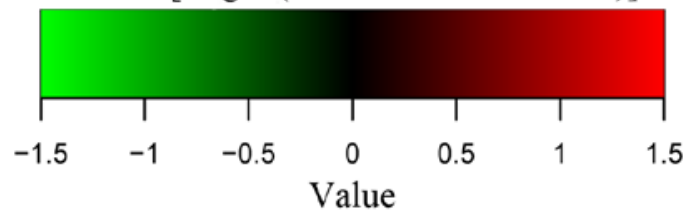

Salivary secretion - Homo sapiens (human)

Gastric acid secretion - Homo sapiens (human)

cAMP signaling pathway - Homo sapiens (human)

Adrenergic signaling in cardiomyocytes - Homo sapiens (human)

cGMP-PKG signaling pathway - Homo sapiens (human)

Pancreatic secretion - Homo sapiens (human)

Bile secretion - Homo sapiens (human)

Proximal tubule bicarbonate reclamation - Homo sapiens (human)

TGF-beta signaling pathway - Homo sapiens (human)

HTLV-I infection - Homo sapiens (human)

Rap1 signaling pathway - Homo sapiens (human)

Alcoholism - Homo sapiens (human)

Transcriptional misregulation in cancer - Homo sapiens (human)

Complement and coagulation cascades - Homo sapiens (human)

Parkinsons disease - Homo sapiens (human)

Oxidative phosphorylation - Homo sapiens (human)

Malaria - Homo sapiens (human)

Figure 7. Heatmaps obtained from KEGG enrichment-based cluster analysis, which shown pathway of alcoholism - Homo sapiens (human), salivary secretion - Homo sapiens (human), malaria - Homo sapiens (human), Parkinson's disease - Homo sapiens (human), oxidative phosphorylation - Homo sapiens (human) were meaningful pathways in this process. KEGG, Kyoto Encyclopedia of Genes and Genomes. Y represents recurrent nasopharyngeal carcinoma (rNPC); N represents non-recurrent nasopharyngeal carcinoma (nrNPC).

in only 49 patients $(29 \mathrm{rNPC}+20 \mathrm{nrNPC})$. The difference of the constituent ratio [3/29 (rNPC) vs. 22/42 (nrNPC)] was significant $(\mathrm{P}=0.000)$, which expressed that $\mathrm{rNPC}$ serums were more easily to be tested for CALM. These data suggest thatCALM may be a promising and useful protective marker for patients with rNPC.

\section{Discussion}

A tremendous challenge in rNPC is the lack of tools which can be used for the early diagnosis of the disease. However, imaging, such as CT, MRI or PET-CT has limited accuracy and cannot confirm the nature of the lesion. Moreover, biopsy is an invasive approach with some side-effects. This results in some patients being diagnosed at a late stage. A non-invasive blood-based test will be a revolutionary step in tumor diagnosis.

In this study, we conducted comprehensive quantitative proteomics analysis to identify a promising biomarker which can be further studied as a non-invasive test for the diagnosis of rNPC. We performed the study in biological replicates.

In two of these, we identified 94 and 83 proteins that were significantly dysregulated in rNPC compared to nrNPC, 


\section{A Downregulated Upregulated}

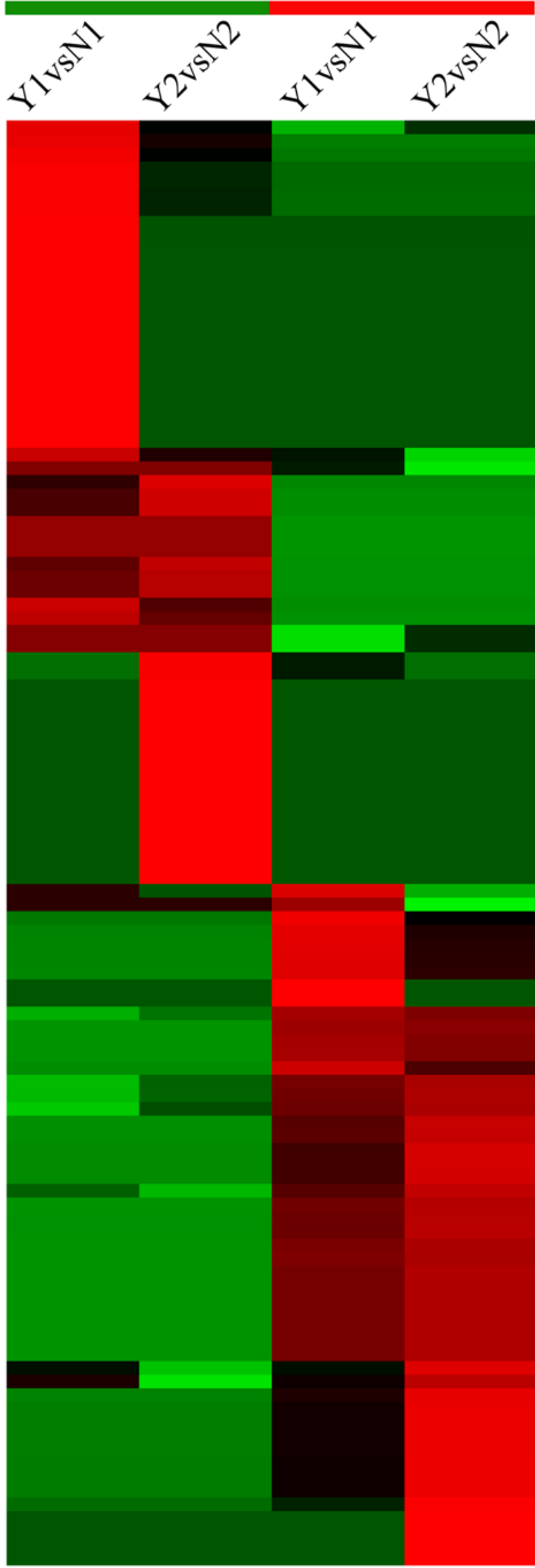

\section{Zscore - $\log 10$ (Fisher's exact P-value)}

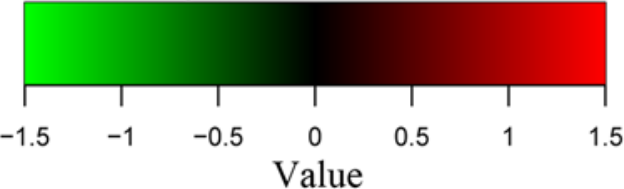

response to metal ion

positive regulation of catalytic activity

regulation of cell cycle process

maintenance of location

response to monosaccharide stimulus

regulation of transmem

regulation of cell cycle
regulation of hydrolase activity

response to carbohydracte stimuly

regulation of meiotic cell cycle

regulation of antigen processing and presentation

regulation of dendritic cell antigen processing and presentatio

regulation of antigen processing and presentation of peptide or polysaccharide antigen via MHC class II negative regulation of antigen processing and presentation

Golgi ribbon formation

membrane invagination

negative regulation of molecular function

cellular response to heat

regulation of catalytic activity

negative regulation of catalytic activity

cellular senescence

negative regulation of transpo

response to magnesium io

mplex assembly

acute inflammatory response

vesicle-m
endocytosis

single-organism transport

negative regulation of lipid storage

actin filament-based process

secretion

establishment of localization

response to calcium ion

establishment of localization in cell

muscle contraction

muscle system proces

regulation of protein depolymerization

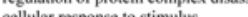

cellular response to

axis specificatio

carbohydrate derivative transport

multivesicular body sorting pathway

neurotrophin production

rufle organization
regulation of integrin activation

signaling

nerve growth factor production

cell communication
signal transduction

positive regulation of respiratory burs

detection of chemical stimulus

single organism signaling

inflammatory response

response to wounding

chromosome organis ossifin

chromatin organization

protein-DNA complex subunit organization

regulation of epithelial cell proliferation

response to stress

negative regulation of multicellular organismal process

protein activation cascad

hydrogen transport

pen peroxide

hydrogen peroxide metabolic process

defense response

positive regulation of immune response

humoral immune response

positive regulation of response to stimules

reactive oxygen species metabolic process

renal system process

vascular process in circulatory system

regulation of immune system process

immune respons

regulation of immune response

cellular response to oxidative stres

innate immune response

response to oxidative stress

pasitive ro rative a yen species

adaptive immune response based on somatic recombination of immune receptors built from immunoglobulin superfamily domains lymphocyte mediated immunity

B cell mediated immunity

leukocyte mediated immunity

adaptive immune response

response to alcohol

response to inorganic substance

positive regulation of cell deat

porphyrin-containing compound metabolic process

male sex differentiation

fibril organization

negative regulation of tissue remodelin

pigment metabolic process

embryo implantatio

destlopment of primary sexual

列

response to oxygen-containing compous

regulation of organ growth

Figure 8. (A) Heatmaps obtained from biological process enrichment-based cluster analysis, which show antigen binding, proton-transporting ATPase activity, rotational mechanism, hydrogen ion transmembrane transporter activity and MHC class I protein binding processes were the highest enrichment parts. 

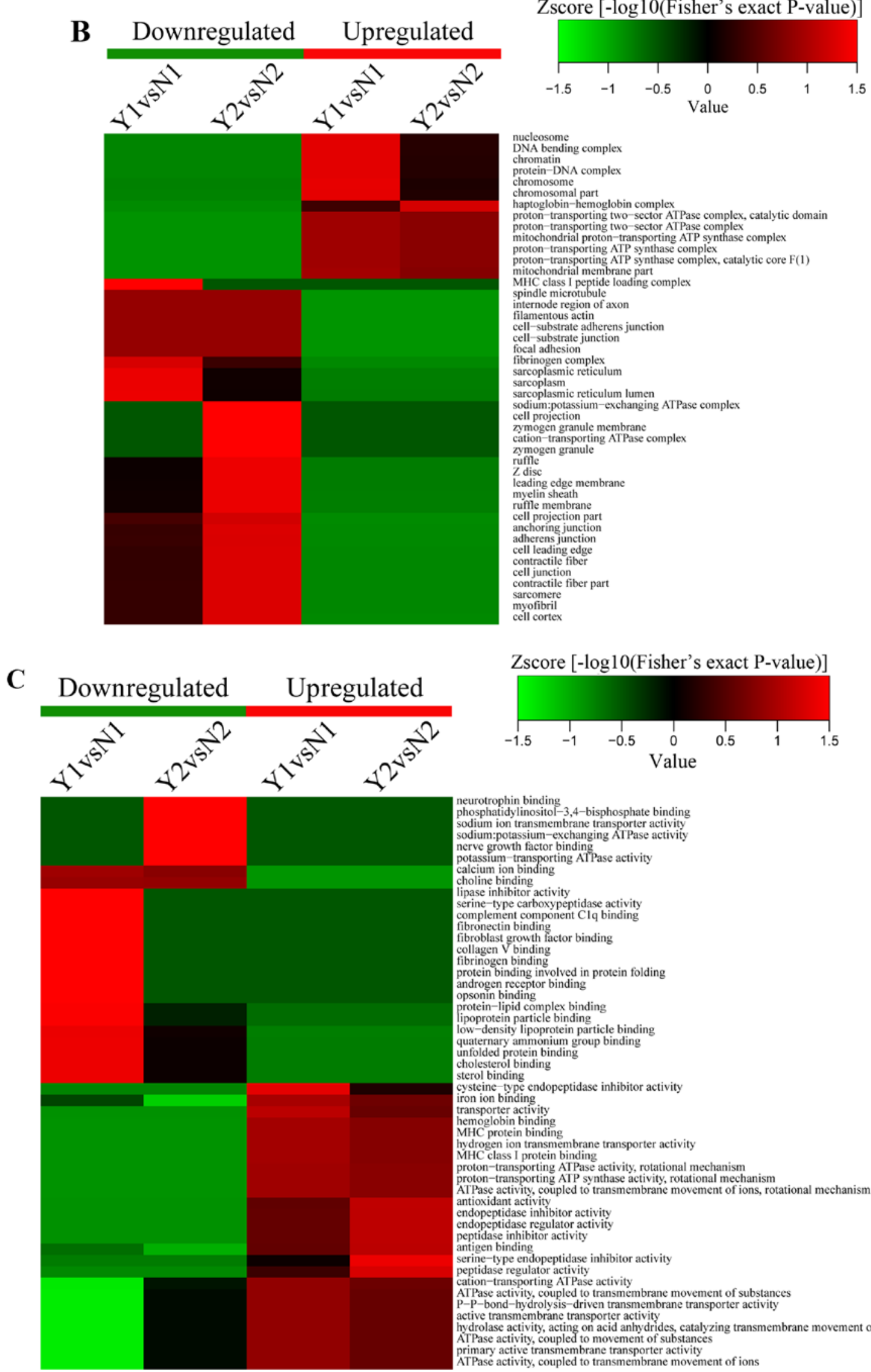

neurotrophin binding
phosphatidylinositol-3,4-bisphosphate binding sodium ion transmembrane transporter activity sodium:potassium-exchanging ATPase activity nerve growth factor binding potassium-transporting ATPase activity calcium ion binding lipase inhibitor activity serine-type carboxypeptidase activity complement component $\mathrm{Clq}$ binding fibronectin binding
fibroblast growth factor binding
collagen $V$ binding collagen $v$ binding protein binding involved in protein folding androgen receptor binding opsonin binding protein-lipid complex bindin lipoprotein particle binding oprotein particle binding quaternary ammonium group binding unfolded protein binding cholesterol binding sterol binding
cysteine-type

cysteine type endopeptidase inhibitor activity transporter activity hemoglobin binding MHC protein binding

hydrogen ion transmembrane transporter activity MHC class I protein binding

proton-transporting ATPase activity, rotational mechanism

ATPase activity, coupled to transmembrane movement of ions, rotational mechanism

endopeptidase inhibitor activity

endopeptidase regulator activity

peptidase inhibitor activity

serine-type en

peptidase regulator activity

cation-transporting ATPase activity

ATPase activity, coupled to transmembrane movement of substances

P-P-bond-hydrolysis-driven transmembrane transporter activity

hydrolase activity acting on acid anhydrid

Catyzing transmembrane movement of substances coupled to movement of substances

ATPase activity, coupled to transmembrane movement of ions

Figure 8. Continued. (B) Heatmaps obtained from cellular component enrichment-based cluster analysis, which show nucleosome, DNA bending complex, chromatin, protein-DNA complex, proton-transporting two-sector ATPase complex and ATPase activity, coupled to transmembrane movement of ions, and rotational mechanism were the meaningful enrichment parts. (C) Heatmaps obtained from molecular function enrichment-based cluster analysis, which show function of proton-transporting ATPase activity, rotational mechanism, ATPase activity, coupled to transmembrane movement of ions, rotational mechanism, heme transporter activity, protein transmembrane transporter activity and isocitrate hydro-lyase (cis-aconitate-forming) activity were the meaningful enrichment parts. Y represents recurrent nasopharyngeal carcinoma (rNPC); $\mathrm{N}$ represents non-recurrent nasopharyngeal carcinoma (nrNPC). 

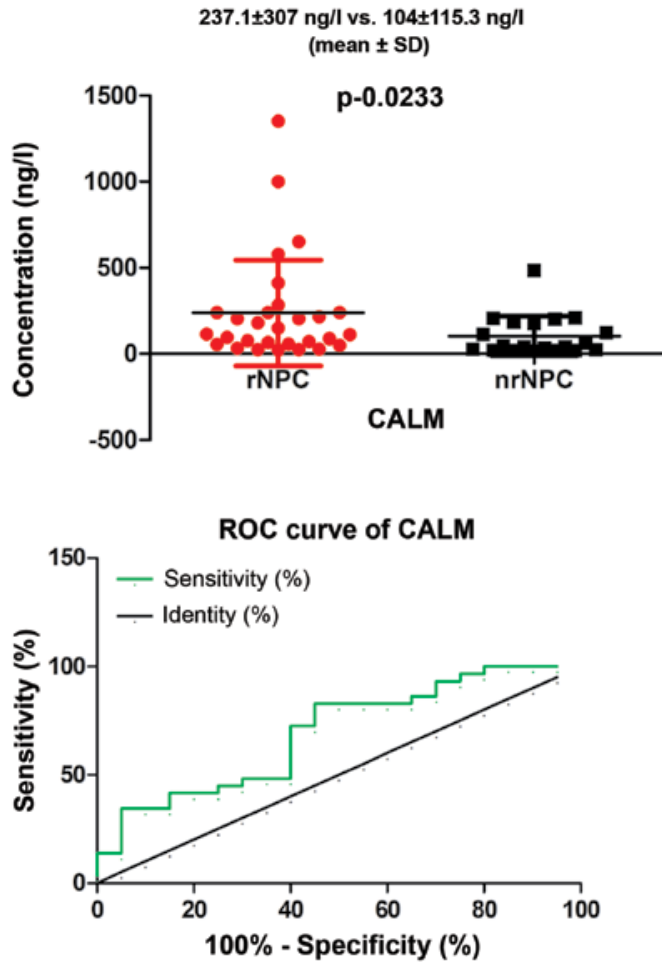

Figure 9. ROC curve of CALM proteins, the area of CALM is 0.6931 , $\mathrm{P}=0.02275$, which showed CALM got some power in clininal used. Compared to nrNPC, the concentration of CALM was higher in rNPC significantly. CALM, calmodulinis; nrNPC, no recurrence nasopharyngeal carcinoma; rNPC, recurrent nasopharyngeal carcinoma.

respectively. Intensive bioinformatics analysis was carried out to annotate these quantifiable targets, including protein annotation, functional classification, functional enrichment, functional enrichment-based cluster analysis, etc.

There are some unique aspects in the present study. The first one, was that we performed biological replicates. Moreover, the data of two trials receied a low heterogeneity (Fig. 10), which showed a high confidence in LC-MS/MS analysis. In addition, we used TMT labeling which allows for the simultaneous accurate, reproducible and precise quantification of proteins across complex samples, which is considered superior to two-dimensional liquid chromatographic approach and iTRAQ $(14,17)$. It offered a more confident expression in our study.

Furthermore, we did not find a related report about rNPC patient serum using TMT labeling, which meant that, at least to the best of our knowledge, this was the first test comparing the serum of patients with rNPC and nrNPC in a high throughput approach.

It is known that NPC is a multifactorial disease, including host genetics, chronic infection by EBV, environmental factors and others (18). Even after effective treatment, there are still $20 \%$ patients who suffer from recurrence; a well known cause of rNPC is radioresistance (19). The elucidation of the molecular mechanisms and the potential pathways involved in rNPC carcinogenesis can certainly aid in the diagnosis and prognosis.

Of 59 dysregulated proteins, most proteins have been reported to be connected with tumorigenesis. In our study, CALM was enriched in 4 pathways, the fold change was 2.11 and the enrichment ratio was the highest. In addition, CALM has been reported

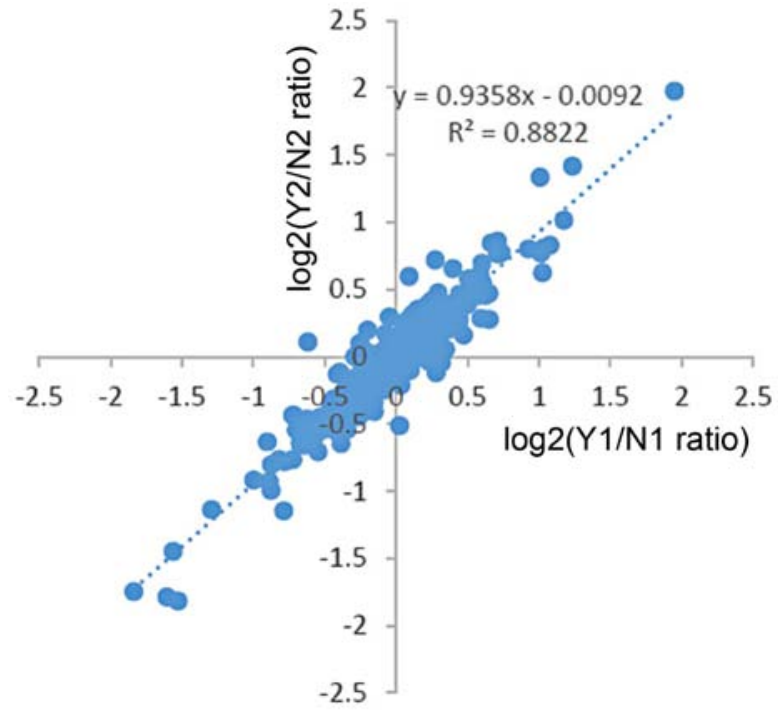

Figure 10. Reproducibility analysis of 2 repeated trials by scatter diagram. The $\mathrm{x}$-axis presents the $\log 2(\mathrm{Y} / \mathrm{N} 1$ ratio) value and the $\mathrm{y}$-axis presents the $\log 2(\mathrm{Y} 2 / \mathrm{N} 2$ ratio) value.Y represents recurrent nasopharyngeal carcinoma (rNPC); N represents non-recurrent nasopharyngeal carcinoma (nrNPC).

to be dysregulated in other malignancies (20-23). All the above suggests that CALM plays a singificant role in rNPC.

To date, it is well known that CALM is considered to be the major regulator of $\mathrm{Ca}^{2+}$-dependent signaling in all eukaryotic cells, which is considered the major regulator of many physiological processes, such as cell proliferation, autophagy and apoptotic processes (24). A previous study demonstrated that compared to normal mouse liver, the concentration of CALM was high in hepatomas (20); others have demonstrated that CALM level was significantly higher in human primary lung cancer (25), and in human T lymphocytes (23) than in normal subjects. A positive correlation between the degree of tumor malignancy with the level of cellular CALM has been published $(20,21)$. Furthermore, there are multiple CALM inhibitors that have been used to inhibit tumor cell growth in different species either in vivo or in culture, such as breast adenocarcinomas (21), lung carcinomas (26), colon carcinomas (27), leukemia cells (28) and resistant pancreatic cancer (29). According to our results, the CALM level was significantly higher in rNPC serum than in nrNPC serum, which suggests that the upregulation of CALM can be a potential biomarker. Even though the association between NPC or rNPC and CALM has not yet been reported, our data suggest that it plays an important role in rNPC; however, this needs to be further confirmed in a larger cohort of patients.

Interestingly, CALM is involved in the Rapl signaling pathway, cAMP signaling pathway and cGMP-PKG signaling pathway processes, even though the enrichment ratios of the above pathways were not high $(\mathrm{P}>0.05)$, which may still demonstrate some connective associations. Several studies have also demonstrated that under hypoxic conditions, the $\mathrm{Ca}^{2+} / \mathrm{CALM}$ complex increases, and it lead to an improvement in the expression of vascular endothelial growth factor and the transcriptional activity of hypoxia-inducible factor 1. CALM antagonists can inhibit the above process (30-32). As we all know, hypoxia is a reason for the radioresistance of NPC, which suggests that 
CALM may be closely associated with rNPC. Further studies are warranted for elucidate to provide more insight into this.

There are limits to the present study. Firstly, we used pooled samples for TMT profiling, which is sensitive to outliers. Thus, it will possible that this may have lead to false positives. This is the reason why we undertook a validation using individual samples. Secondly, we stored the serum at $-80^{\circ} \mathrm{C}$, while we could not collect the sample at the same time, this may lead to the loss of some vital proteins. Finally, as mentioned above, further verifications are required with a large sample size. If the serum volume of each sample was large enough, a PCR study should have also been carried out. This may be an approach with which to elucidate the potential mechanisms responsible for rNPC.

In conclusion, CALM may be a potential biomarker which may greatly improve the prediction and management of rNPC.

\section{Acknowledgements}

The present study was supported by grants from the Health Department of Guangxi Province of China (no. 2012091).

\section{References}

1. Chen ZT, Liang ZG and Zhu XD: A review: Proteomics in nasopharyngeal carcinoma. Int J Mol Sci 16: 15497-15530, 2015.

2. Wei YS, Zheng YH, Liang WB, Zhang JZ, Yang ZH, Lv ML, Jia $J$ and Zhang L: Identification of serum biomarkers for nasopharyngeal carcinoma by proteomic analysis. Cancer 112 : 544-551, 2008

3. Du C, Ying H, Zhou J, Hu C and Zhang Y: Experience with combination of docetaxel, cisplatin plus 5-fluorouracil chemotherapy, and intensity-modulated radiotherapy for locoregionally advanced nasopharyngeal carcinoma. Int J Clin Oncol 18 464-471, 2013

4. Tao YL, Li Y, Gao J, Liu ZG, Tu ZW, Li G, Xu BQ, Niu DL, Jiang CB and Yi W: Identifying FGA peptides as nasopharyngeal carcinoma-associated biomarkers by magnetic beads. J Cell Biochem 113: 2268-2278, 2012.

5. Zhong Yu LJ: Diagnostic progress of recurrent nasopharyngeal carcinoma. Clinical Imaging Technology 24: 1633-1674, 2009.

6. Sahu A, Nandakumar N, Sawant S and Krishna CM: Recurrence prediction in oral cancers: A serum Raman spectroscopy study. Analyst 140: 2294-2301, 2015.

7. Nicholson BD, Shinkins B, Pathiraja I, Roberts NW, James TJ, Mallett S, Perera R, Primrose JN and Mant D: Blood CEA levels for detecting recurrent colorectal cancer. Cochrane Database Syst Rev 12: CD011134, 2015.

8. Li GP, Wang H, Lai YK, Chen SC, Lin MC, Lu G, Zhang JF, He XG, Qian CN and Kung HF: Proteomic profiling between CNE-2 and its strongly metastatic subclone S-18 and functional characterization of HSP27 in metastasis of nasopharyngeal carcinoma. Proteomics 11: 2911-2920, 2011.

9. Xiao Z, Li G, Chen Y, Li M, Peng F, Li C, Li F, Yu Y, Ouyang Y, Xiao Z and Chen Z: Quantitative proteomic analysis of formalin-fixed and paraffin-embedded nasopharyngeal carcinoma using iTRAQ labeling, two-dimensional liquid chromatography, and tandem mass spectrometry. J Histochem Cytochem 58: 517-527, 2010.

10. Seriramalu R, Pang WW, Jayapalan JJ, Mohamed E, Abdul-Rahman PS, Bustam AZ, Khoo AS and Hashim OH: Application of champedak mannose-binding lectin in the glycoproteomic profiling of serum samples unmasks reduced expression of alpha- 2 macroglobulin and complement factor B in patients with nasopharyngeal carcinoma. Electrophoresis 31: 2388-2395, 2010.

11. Tang CE, Tan T, Li C, Chen ZC, Ruan L, Wang HH, Su T, Zhang PF and Xiao ZQ: Identification of Galectin-1 as a novel biomarker in nasopharyngeal carcinoma by proteomic analysis. Oncol Rep 24: 495-500, 2010.
12. Cho WC, Yip TT, Yip C, Yip V, Thulasiraman V, Ngan RK, Yip TT, Lau WH, Au JS and Law SC: Identification of serum amyloid A protein as a potentially useful biomarker to monitor relapse of nasopharyngeal cancer by serum proteomic profiling. Clin Cancer Res 10: 43-52, 2004.

13. Yang X, Dai W, Kwong DL, Szeto CY, Wong EH, Ng WT, Lee AW, Ngan RK, Yau CC and Tung SY: Epigenetic markers for non-invasive early detection of nasopharyngeal carcinoma by methylation-sensitive high resolution melting. Int J Cancer 136: 127-135, 2015.

14. White NM, Masui O, Desouza LV, Krakovska O, Metias S, Romaschin AD, Honey RJ, Stewart R, Pace K, Lee J, et al: Quantitative proteomic analysis reveals potential diagnostic markers and pathways involved in pathogenesis of renal cell carcinoma. Oncotarget 5: 506-518, 2013.

15. Tian X, Sun D, Zhao S, Xiong H and Fang J: Screening of potential diagnostic markers and therapeutic targets against colorectal cancer. Onco Targets Ther 8: 1691-1699, 2015.

16. Yan LR, Wang DX, Liu H, Zhang XX, Zhao H, Hua L, Xu P and Li YS: A pro-atherogenic HDL profile in coronary heart disease patients: an iTRAQ labelling-based proteomic approach. PloS One 9: e98368, 2014.

17. Sandberg A, Branca RM, Lehtiö J and Forshed J: Quantitative accuracy in mass spectrometry based proteomics of complex samples: The impact of labeling and precursor interference. J Proteomics 96: 133-144, 2014.

18. Janvilisri T: Omics-based identification of biomarkers for nasopharyngeal carcinoma. Dis Markers 2015: 762128, 2015.

19. Luftig M: Heavy LIFting: Tumor promotion and radioresistance in NPC. J Clin Invest 123: 4999-5001, 2013.

20. Uenishi K, Criss WE and Kakiuchi S: Calcium-activatable phosphodiesterase and calcium-dependent modulator protein in transplantable hepatoma tissues. J Biochem 87: 601-607, 1980.

21. Wei JW, Morris HP and Hickie RA: Positive correlation between calmodulin content and hepatoma growth rates. Cancer Res 42 : 2571-2574, 1982.

22. Colomer J, Agell N, Engel P and Bachs O: Expression of calmodulin and calmodulin binding proteins in lymphoblastoid cells. J Cell Physiol 159: 542-550, 1994.

23. Colomer J, Agell N, Engel P, Alberola-Ila J and Bachs O: Calmodulin expression during proliferative activation of human T lymphocytes. Cell calclum 14: 609-618, 1993.

24. Berchtold MW and Villalobo A: The many faces of calmodulin in cell proliferation, programmed cell death, autophagy, and cancer. Biochim Biophys Acta 1843: 398-435, 2014.

25. Liu GX, Sheng HF and Wu S: A study on the levels of calmodulin and DNA in human lung cancer cells. Br J Cancer 73: 889-901, 1996.

26. Schuller HM, Correa E, Orloff M, Reznik GK: Successful chemotherapy of experimental neuroendocrine lung tumors in hamsters with an antagonist of $\mathrm{Ca}^{2+} /$ calmodulin. Cancer Res 50: 1645-1649, 1990.

27. Lönn U and Lönn S: Increased growth inhibition and DNA lesions in human colon adenocarcinoma cells treated with methotrexate or 5-fluorodeoxyuridine followed by calmodulin inhibitor. Cancer Res 48: 3319-3323, 1988.

28. Hait WN, Gesmonde J and Cheng E: Effects of KS-501, KS-502 and their enantiomers on calmodulin-sensitive enzyme activity and cellular proliferation. Biochem Pharmacol 50: 69-74, 1995.

29. Yuan K, Yong S, Xu F, Zhou T, McDonald JM and Chen Y: Calmodulin antagonists promote TRA-8 therapy of resistant pancreatic cancer. Oncotarget 6: 25308-25319, 2015.

30. Mukhopadhyay D and Akbarali HI: Depletion of $\left[\mathrm{Ca}^{2+}\right]$ i inhibits hypoxia-induced vascular permeability factor (vascular endothelial growth factor) gene expression. Biochem Biophys Res Commun 229: 733-738, 1996.

31. Salnikow K, Kluz T, Costa M, Piquemal D, Demidenko ZN, Xie $\mathrm{K}$ and Blagosklonny MV: The regulation of hypoxic genes by calcium involves c-Jun/AP-1, which cooperates with hypoxia-inducible factor 1 in response to hypoxia. Mol Cell Biol 22: 1734-1741, 2002.

32. Jung HJ, Kim JH, Shim JS and Kwon HJ: A novel $\mathrm{Ca}^{2+} /$ calmodulin antagonist $\mathrm{HBC}$ inhibits angiogenesis and downregulates hypoxia-inducible factor. J Biol Chem 285: 25867-25874, 2010. 